\title{
Video Article \\ Screening for Amyloid Aggregation by Semi-Denaturing Detergent-Agarose Gel Electrophoresis
}

\author{
Randal Halfmann ${ }^{1,2,3}$, Susan Lindquist ${ }^{1,2,3}$ \\ ${ }^{1}$ Whitehead Institute for Biomedical Research \\ ${ }^{2}$ Department of Biology, MIT - Massachusetts Institute of Technology \\ ${ }^{3}$ Howard Hughes Medical Institute
}

Correspondence to: Susan Lindquist at lindquist_admin@wi.mit.edu

URL: https://www.jove.com/video/838

DOI: doi:10.3791/838

Keywords: Basic Protocols, Issue 17, biochemistry, SDD-AGE, amyloid, prion, aggregate

Date Published: $7 / 16 / 2008$

Citation: Halfmann, R., Lindquist, S. Screening for Amyloid Aggregation by Semi-Denaturing Detergent-Agarose Gel Electrophoresis. J. Vis. Exp. (17), e838, doi:10.3791/838 (2008)

\section{Abstract}

Amyloid aggregation is associated with numerous protein misfolding pathologies and underlies the infectious properties of prions, which are conformationally self-templating proteins that are thought to have beneficial roles in lower organisms. Amyloids have been notoriously difficult to study due to their insolubility and structural heterogeneity. However, resolution of amyloid polymers based on size and detergent insolubility has been made possible by Semi-Denaturing Detergent-Agarose Gel Electrophoresis (SDD-AGE). This technique is finding widespread use for the detection and characterization of amyloid conformational variants. Here, we demonstrate an adaptation of this technique that facilitates its use in large-scale applications, such as screens for novel prions and other amyloidogenic proteins. The new SDD-AGE method uses capillary transfer for greater reliability and ease of use, and allows any sized gel to be accomodated. Thus, a large number of samples, prepared from cells or purified proteins, can be processed simultaneously for the presence of SDS-insoluble conformers of tagged proteins.

\section{Video Link}

The video component of this article can be found at https://www.jove.com/video/838/

\section{Protocol}

\section{Part 1: Preparing the gel}

1. Assemble the gel casting tray. Standard equipment for horizontal DNA electrophoresis can be used. For large numbers of samples, we prepare a $20 \mathrm{~cm} \times 24 \mathrm{~cm}$ slab with up to four 50-well combs. Make sure the slab does not have scratches, as these can distort the blot image.

2. Create a $1.5 \%$ agarose solution (medium or high gel-strength, low EEO) in $1 \mathrm{X}$ TAE. The volume should be enough to completely submerge the comb teeth -- you will want to load as much sample as possible to maximize detection. Microwave the mixture until the agarose is completely dissolved.

3. Rapidly add SDS to $0.1 \%$ from a $10 \%$ stock. Swirl to mix. If some agarose solidifies during this step, redissolve using a hot plate and be careful to avoid boiling.

4. Pour the solution into the casting tray. Use a comb to rake out any bubbles, as they could later interfere with transfer.

5. After gel has set, remove combs and place the gel into the gel tank. Completely submerge the gel in $1 X$ TAE containing $0.1 \%$ SDS.

\section{Part 2: Preparing samples}

1. For high-throughput analysis of yeast lysates, we start with 2-ml cultures grown overnight with rapid agitation in 96-well blocks. In this case, each culture is overexpressing a protein of interest. When analyzing low abundance proteins, larger culture volumes must be used

2. Harvest cells by centrifugation at $2000 \mathrm{RCF}$ for $5 \mathrm{~min}$ at room temperature.

3. Resuspend cells in water and centrifuge again.

4. Resuspend in $1 \mathrm{ml}$ spheroplasting solution. Incubate for approximately $30 \mathrm{~min}$ at $30^{\circ} \mathrm{C}$ (you may confirm spheroplasting efficiency by microscopy).

5. Centrifuge at $800 \mathrm{RCF}$ for $5 \mathrm{~min}$ at room temperature. Completely remove supernatant.

6. Resuspend pelleted spheroplasts in $100 \mathrm{ml}$ lysis buffer.

7. Cover the block with tape and vortex on high speed for 2 min.

8. Pellet cellular debris at $4000 \mathrm{RCF}$ for $2 \mathrm{~min}$.

9. Carefully remove supernatant to a new container, e.g., a 96-well PCR plate.

10. If desired, determine the protein concentration of the lysates.

11. Add $4 \mathrm{X}$ sample buffer to the samples to generate lysates containing $1 \mathrm{X}$ sample buffer. Incubate for 5 min at room temperature. 
12. Load gel. If desired, save half of the sample volume and boil it for SDS-PAGE analysis. In order to monitor the extent of transfer later on, include a pre-stained SDS-PAGE marker. Additionally, a molecular weight marker consisting of very large proteins (e.g., chicken pectoralis extract) can be used to estimate sizes of the resolved complexes.

13. Run at low voltage ( $\leq 3 \mathrm{~V} / \mathrm{cm}$ gel length) until the dye front reaches $\sim 1 \mathrm{~cm}$ from the end of the gel. This will take several hours. It is important that the gel remain cool; otherwise diffusion of low molecular weight protein (e.g., monomers) can limit their detection.

\section{Part 3: Transfer}

1. Cut a piece of nitrocellulose to the same dimensions as the gel.

2. Cut 20 pieces of GB004 and 8 pieces of GB002 blotting paper, to the same dimensions as the gel. Cut an additional piece of GB004 to be used as a wick; make it about 20 centimeters wider than the gel.

3. Immerse the nitrocellulose, wick, and 4 pieces of GB002 in 1X TBS.

4. In a plastic container, assemble a stack of papers as follows: 20 pieces of dry GB004, then 4 pieces of dry GB002, then one piece of pre-wet GB002. Lay the nitrocellulose on top of this stack.

5. Rinse the gel on the casting tray briefly in water to remove excess running buffer. Then, carefully begin to slide the gel off the tray onto the stack. While sliding the gel off the tray, douse the membrane with TBS as necessary. The extra buffer helps prevent bubbles from becoming trapped under the gel. A transfer pipette works well for this purpose.

6. After the gel has been moved to the stack, check thoroughly for bubbles. If any are present, lift the edge of the gel and reapply buffer until the bubbles can be worked out

7. Put the remaining three pre-wetted GB002 pieces on top of the gel. Ensure thorough contact between all layers by rolling a pipette firmly across the top of the stack.

8. Flank the transfer stack with two elevated trays containing TBS. Drape the pre-wet wick across the stack such that either end of the wick is submerged in TBS.

9. Cover the assembled transfer stack with an additional plastic tray bearing extra weight (e.g., a $500 \mathrm{ml}$ bottle of water).

10. Allow the transfer to proceed for a minimum of three hours, or overnight.

11. After transfer, the membrane can be processed by standard Western blotting.

Spheroplasting Solution

$1.2 \mathrm{M} \mathrm{D}$-sorbitol

$0.5 \mathrm{mM} \mathrm{MgCl} 2$

$20 \mathrm{mM}$ Tris, $\mathrm{pH} 7.5$

$50 \mathrm{mM}$ BME (add fresh)

$0.5 \mathrm{mg} / \mathrm{ml}$ Zymolyase 100T (add fresh)

Lysis Buffer

$100 \mathrm{mM}$ Tris 7.5

$50 \mathrm{mM} \mathrm{NaCl}$

$10 \mathrm{mM}$ BME (add fresh)

protease inhibitors (add fresh)

4X Sample Buffer

2X TAE

$20 \%$ glycerol

$8 \%$ SDS

bromophenol blue to preference

\section{Discussion}

SDD-AGE was first reported by Kryndushkin et al. ${ }^{1}$, to study SDS-resistant complexes of the [PSI+] prion in yeast, and has since found widespread use studying both prion and non-prion aggregates ${ }^{2-9}$. However, transfer of the proteins to a membrane following electrophoresis in an agarose gel is problematic, and can result in a distorted blot image ${ }^{5}$. Additionally, the submerged electroblotting technique most commonly used introduces practical limitations for the size of the gel and thus the number of samples that can be processed. We have addressed these problems by employing downward capillary transfer ${ }^{10}$, a simple procedure which uses a stack of dry blotting papers to transfer proteins from the gel to a nitrocellulose membrane. Capillary transfer prevents distortion and allows large gels to be processed easily. There are a few things to consider before using SDD-AGE. For crude samples (e.g., lysates), immunodetection of specific proteins is necessary. SDD-AGE does not fully denature the protein complexes of interest, so the protein(s) to be detected must bear an epitope tag outside of the amyloidogenic region. Lysates can generally be prepared as they would be for a normal SDS-PAGE, with two important differences. First, increased care must be taken to prevent degradation by proteolysis. The partially denaturing conditions used here are not sufficient to inactivate proteases, and can also make target proteins more susceptible to proteolysis. Use a complete protease inhibitor cocktail at at least two-fold the recommended concentration. Second, heating the samples should be avoided. If an all-monomer negative control is desired, for instance to confirm that high-molecular-weight species are not due to covalent modifications, a 10-minute incubation at $95^{\circ} \mathrm{C}$ can be used, which will restore most amyloids to monomeric protein.

\section{Acknowledgements}

We thank Simon Alberti for assistance with developing this protocol. This work was supported by a grant from the National Institutes of Health (GM25874), a Howard Hughes Medical Institute Investigatorship (to S.L.), and a National Science Foundation predoctoral training grant (to R.H.). 


\section{References}

1. Kryndushkin, D.S., Alexandrov, I.M., Ter-Avanesyan, M.D. \& Kushnirov, V.V. Yeast [PSI+] prion aggregates are formed by small Sup35 polymers fragmented by Hsp104. J. Biol. Chem. 278, 49636-49643 (2003).

2. Alexandrov, I.M., Vishnevskaya, A.B., Ter-Avanesyan, M.D. \& Kushnirov, V.V. Appearance and Propagation of Polyglutamine-based Amyloids in Yeast: TYROSINE RESIDUES ENABLE POLYMER FRAGMENTATION. J. Biol. Chem. 283, 15185-15192 (2008).

3. Allen, K.D. et al. Hsp70 chaperones as modulators of prion life cycle: novel effects of Ssa and Ssb on the Saccharomyces cerevisiae prion [PSI + ]. Genetics 169, 1227-1242 (2005).

4. Aron, R., Higurashi, T., Sahi, C. \& Craig, E.A. J-protein co-chaperone Sis1 required for generation of [RNQ+] seeds necessary for prion propagation. The EMBO journal 26, 3794-3803 (2007).

5. Bagriantsev, S.N., Kushnirov, V.V. \& Liebman, S.W. Analysis of amyloid aggregates using agarose gel electrophoresis. Methods in Enzymology 412, 33-48 (2006).

6. Borchsenius, A.S., Muller, S., Newnam, G.P., Inge-Vechtomov, S.G. \& Chernoff, Y.O. Prion variant maintained only at high levels of the Hsp104 disaggregase. Current Genetics 49, 21-29 (2006).

7. Salnikova, A.B., Kryndushkin, D.S., Smirnov, V.N., Kushnirov, V.V. \& Ter-Avanesyan, M.D. Nonsense suppression in yeast cells overproducing Sup35 (eRF3) is caused by its non-heritable amyloids. J. Biol. Chem. 280, 8808-8812 (2005).

8. Tank, E.M., Harris, D.A., Desai, A.A. \& True, H.L. Prion protein repeat expansion results in increased aggregation and reveals phenotypic variability. Mol. Cell. Biol. 27, 5445-5455 (2007).

9. Douglas, P.M. et al. Chaperone-dependent amyloid assembly protects cells from prion toxicity. Proc. Natl. Acad. Sci. USA 105, 7206-7211 (2008).

10. Nagy, B., Costello, R., and Csako, G. Downward blotting of proteins in a model based on apolipoprotein(a) phenotyping. Analytical Biochemistry. 231, 40-45 (1995). 